\title{
LEBESGUE SPACE ESTIMATES FOR A CLASS OF FOURIER INTEGRAL OPERATORS ASSOCIATED WITH WAVE PROPAGATION
}

\author{
SANGHYUK LEE ANDREAS SEEGER \\ Dedicated to Professor Hans Triebel
}

\begin{abstract}
We prove $L^{q}$ estimates related to Sogge's conjecture for a class of Fourier integral operators associated with wave equations.
\end{abstract}

\section{INTRODUCTION}

In this note we prove a variable coefficient version of a recent result in [6] on the local $L^{q}$ space-time regularity results for solutions of wave equations. The solution operators are Fourier integral operators satisfying the 'cinematic curvature' hypothesis introduced in [17] (see also [14]).

For the general setup let $y$ and $z$ be paracompact $C^{\infty}$ manifolds, $\operatorname{dim}(y)=d, \operatorname{dim}(z)=$ $d+1$; in the current paper we shall need to assume $d \geq 4$. We are interested in sharp local regularity estimates for Fourier integral operators $\mathcal{F} \in I^{\mu-1 / 4}(z, y ; \mathcal{C})$ (associated with the Fourier integral distributions defined in $[8])$. Here the canonical relation

$$
\mathcal{C} \subset T^{*} z \backslash 0_{L} \times T^{*} y \backslash 0_{R}
$$

is a conic manifold of dimension $2 d+1$, which is Lagrangian with respect to the symplectic form $d \zeta \wedge d z-d \eta \wedge d y$. We denote by $0_{L}$ and $0_{R}$ the zero-sections in $T^{*} z$ and $T^{*} y$, respectively.

We formulate a curvature hypothesis which appeared in [3], [10] for classes of oscillatory integral operators (see [13], [2] for current results on these classes). We follow the exposition in [14] and impose conditions on the following projection maps.

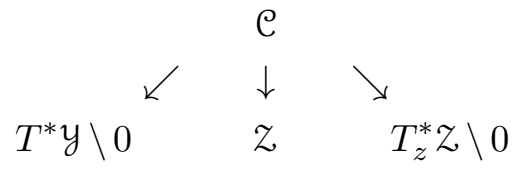

We require that the projection $\pi_{L}: \mathcal{C} \rightarrow T^{*} y$ is a submersion (i.e. the differential has maximal rank $2 d$ ). We also require that the space projection $\Pi_{\mathcal{Z}}: \mathcal{C} \rightarrow \mathcal{Z}$ is a submersion (i.e. its differential has maximal rank $d+1$ ). As discussed in $\S 2$ of [14] this implies that for fixed $z \in \Pi_{Z} \mathrm{C}$ the image of the projection to the fiber,

$$
\Gamma_{z}=\left\{\zeta: \in T_{z}^{*} z: \exists(y, \eta) \text { such that }(z, \zeta, y, \eta) \in \mathcal{C}\right\}
$$

Date: July 27, 2012.

Key words and phrases. Space-time estimates for wave equations on compact manifolds, cinematic curvature, Fourier integral operators.

Supported in part by NRF grant 2011-0001251 (Korea) and and NSF grant 0652890 (USA). 
is an immersed conic $(d-1)$-dimensional hypersurface in $T_{z}^{*} z \backslash 0_{L}$. We then make an assumption on the curvature of the cones $\Gamma_{z}$ :

Curvature hypothesis $\mathcal{H}(\ell): \mathcal{C} \subset T^{*} \mathcal{Z} \backslash 0_{L} \times T^{*} y \backslash 0_{R}$, the projections $\pi_{R}$ and $\Pi_{z}$ are submersions and for each $z$ the cone $\Gamma_{z}$ has at least $\ell$ nonvanishing principal curvatures at any $\zeta \in \Gamma_{z}$.

Theorem 1.1. Let $\ell \geq 3$, let $\mathcal{C}$ satisfy hypothesis $\mathcal{H}(\ell)$ and let $\mathcal{F} \in I^{\mu-1 / 4}(\mathcal{Z}, y ; \mathcal{C})$. Suppose $\frac{2 \ell}{\ell-2}<q<\infty$ and $\mu \leq \frac{d}{q}-\frac{d-1}{2}$. Then $\mathcal{F}$ maps $L_{\text {comp }}^{q}(y)$ to $L_{\mathrm{loc}}^{q}(Z)$.

We may apply the theorem with $\ell=d-1$ to solutions of the wave equation on a compact Riemannian manifold $M$, with initial data in $L^{q}$-Sobolev spaces $L_{\alpha}^{q}(M)$. Let $\Delta$ be the Laplace-Beltrami operator on $M$. If one combines Theorem 1.1 with the usual parametrix construction ( $c f .[4]$ ) one obtains (arguing as in [14])

Corollary 1.2. Let $d \geq 4, \frac{2(d-1)}{d-3}<q<\infty$, and let $I$ be a compact time interval. There is $C>0$ such that

$$
\left(\int_{I}\left\|e^{i t \sqrt{-\Delta}} f\right\|_{L^{q}(M)}^{q} d t\right)^{1 / q} \leq C\|f\|_{L_{\alpha}^{q}(M)}, \quad \alpha=\frac{d-1}{2}-\frac{d}{q},
$$

for all $f \in L_{\alpha}^{q}(M)$.

Note that the constant may strongly depend on the choice of $I$. There are further regularity improvements in the scale of Triebel-Lizorkin spaces ( $c f . \S 3$ below); in particular $L_{\alpha}^{q}(M)$ can be replaced by the Besov space $B_{\alpha, q}^{q}(M)$.

In $\S 2$ we prove a frequency localized version of Theorem 1.1 and combine the estimates corresponding to different frequencies in $\S 3$. In $\S 4$ we discuss some generalizations in the constant coefficient case.

Remarks. In the constant coefficient case one can recover from Theorem 1.1 the space time estimates of [6] which correspond to an endpoint version of Sogge's conjecture in the range given for $\ell=d-1$, see also $\S 4$ for other generalizations. For previous partial results on Sogge's conjecture, also in lower dimensions, see the groundbreaking paper of Wolff [20] and the subsequent papers [12], [5].

The case $\ell=d-1$ essentially corresponds to the assumption of cinematic curvature in [17]. We use Hörmander's convention for the definition of order, i.e., in view of the different dimensions of $z$ and $y$ operators of class $I^{\mu-1 / 4}(z, y ; \mathcal{C})$ correspond to locally finite sums of operators with integral kernels in the standard representation (1) below, involving $d$ frequency variables and standard symbols of order $\mu$. One can use a partition of unity and finite decompositions in the fiber variable to reduce matters to the estimation of an integral operator with compactly supported kernel $\mathcal{K}$ which is given as an oscillatory integral distribution in the sense of [8]. Namely if $Z$ is an open set in $\mathbb{R}^{d+1}$ and $Y$ is an open set in $\mathbb{R}^{d}$ we may assume that

$$
\mathcal{K}(z, y)=\int a(z, y, \xi) e^{i(\phi(z, \xi)-\langle y, \xi\rangle)} d \xi
$$

where $a$ is a standard symbol of order $\mu, a$ is supported for $z, y$ in compact subsets of $Z$ and $Y$, resp., and $\phi$ is smooth away from the origin and homogeneous of order one with respect to the variable $\xi$, and supported in an open set which is conic in $\xi$. We then have $\nabla_{z} \phi(z, \xi) \neq 0$ for $\xi \neq 0$ and the mixed second derivative $(d+1) \times d$ matrix $\phi_{z \xi}^{\prime \prime}(z, \xi)$ has rank $d$. For fixed $(z, \xi)$, if the vector $u$ is in the cokernel of $\phi_{z \xi}^{\prime \prime}(z, \xi)$ then the Hessian matrix $\nabla_{\xi \xi}^{2}\left\langle u, \nabla_{z} \phi\right\rangle(z, \xi)$ has rank at least $\ell$, by our curvature assumption. 


\section{The FREQUENCY LOCALIZED CASE}

By making further localizations, changing variables in $z$ and $y$, and ignoring error terms which are smoothing of high order we may assume that our kernel is given by

$$
K(z, y)=\sum_{k=1}^{\infty} 2^{k \mu} K_{k}(z, y),
$$

where

$$
K_{k}(z, y)=\int \chi_{k}\left(z, y, 2^{-k} \xi\right) e^{i(\varphi(z, \xi)-\langle y, \xi\rangle)} d \xi
$$

and the functions $\chi_{k}$ are smooth and supported in a compact subset of $Z \times Y \times \Xi$. Here $Z$ is a small neighborhood of the origin in $\mathbb{R}^{d+1}, Y$ is a small neighborhood of the origin in $\mathbb{R}^{d}$ and $\Xi$ is a small neighborhood of the vector $e_{1}:=(1,0, \ldots, 0)$ in $\mathbb{R}^{d}$. Moreover

$$
\begin{gathered}
\varphi_{z \xi}^{\prime \prime}\left(0, e_{1}\right)=\left(\begin{array}{c}
I_{d} \\
0
\end{array}\right) \\
\operatorname{rank} \nabla_{\xi \xi}^{2} \varphi_{z_{d+1}}^{\prime}\left(0, e_{1}\right) \geq \ell ;
\end{gathered}
$$

and in view of the small choice of $Z, Y, \Xi$ we may assume that for all $(z, \xi) \in Z \times \Xi$ the gradient $\varphi_{z}^{\prime}(z, \xi)$ is close to $e_{1}$, and, with $z=\left(z^{\prime}, z_{d+1}\right)$, we may assume that $\varphi_{z^{\prime} \xi}^{\prime \prime}(z, \xi)$ is close to the identity matrix $I_{d}$ and $\varphi_{z_{d+1} \xi}^{\prime \prime}(z, \xi)$ is small. We may further perform a rotation and assume that in coordinates $\xi=\left(\xi_{1}, \xi^{\prime}, \xi^{\prime \prime}\right)$ with $\xi^{\prime}=\left(\xi_{2}, \ldots, \xi_{\ell+1}\right)$ we have

$$
\operatorname{rank} \nabla_{\xi^{\prime} \xi^{\prime}}^{2} \varphi_{z_{d+1}}^{\prime}\left(0, e_{1}\right)=\ell
$$

Finally, $\left|\partial_{z, y, \xi}^{\alpha} \chi_{k}(z, y, \xi)\right| \leq C_{\alpha}$ for any multiindex $\alpha$, uniformly in $k,(z, y, \xi) \in Z \times Y \times \Xi$.

Let $T_{k} f(z)=\int K_{k}(z, y) f(y) d y$. Here we prove that the $L^{q}\left(\mathbb{R}^{d}\right) \rightarrow L^{q}\left(\mathbb{R}^{d+1}\right)$ operator norm of $T_{k}$ is $O\left(2^{k\left(\frac{d-1}{2}-\frac{d}{q}\right)}\right)$ for $q>\frac{2 \ell}{\ell-2}$, and in the next section we discuss how to put the estimates for $T_{k}$ together. The $L^{\infty}$ estimate

$$
\left\|T_{k} f\right\|_{L^{\infty}} \lesssim 2^{k \frac{d-1}{2}}\|f\|_{\infty}
$$

can be found in [16]. By interpolation it is enough to prove

Theorem 2.1. Let $\ell \geq 3$ and $q_{\ell}=\frac{2 \ell}{\ell-2}$. The operator $T_{k}$ is of restricted weak type $\left(q_{\ell}, q_{\ell}\right)$, with operator norm

$$
\left\|T_{k}\right\|_{L^{q_{\ell}, 1}\left(\mathbb{R}^{d+1}\right) \rightarrow L^{q_{\ell}, \infty}\left(\mathbb{R}^{d}\right)} \lesssim 2^{k(d / \ell-1 / 2)} .
$$

By duality we need to prove the restricted weak type inequality for the adjoint operator $T_{k}^{*}$, given by

$$
T_{k}^{*} F(y)=\iint \chi_{k}\left(z, y, 2^{-k} \xi\right) e^{i(\langle y, \xi\rangle-\varphi(z, \xi))} d \xi F(z) d z ;
$$

i.e. for each measurable set $E$ contained in $[-1 / 2,1 / 2]^{d+1}$,

$$
\left\|T_{k}^{*} \chi_{E}\right\|_{L^{p_{\ell}, \infty}}^{p_{\ell}} \lesssim 2^{k \frac{2 d-\ell}{\ell+2}}|E|, \quad p_{\ell}=\frac{2 \ell}{\ell+2} .
$$

The estimate (6) will be derived from the following Proposition 2.2, which is a discretized version of (6) and will be proved in $\S 2.3$. 
Proposition 2.2. Let $p_{\ell}=\frac{2 \ell}{\ell+2}, \ell \geq 3$. For $k>2$ let $\mathcal{Z}_{k}=2^{-k} \mathbb{Z}^{d+1} \cap\left[-\varepsilon^{2}, \varepsilon^{2}\right]^{d+1}$, for sufficiently small $\varepsilon>0$. Suppose that for each $\mathfrak{z} \in \mathcal{Z}_{k}$ we are given a symbol $a_{k, \mathfrak{z}}$ supported in $\left\{\xi: 2^{k-1}<|\xi|<2^{k+1},\left|\frac{\xi}{|\xi|}-e_{1}\right| \leq \varepsilon^{2}\right\}$ so that

$$
\left|\partial_{\xi}^{\alpha} a_{k, \mathfrak{z}}(\xi)\right| \leq 2^{-k|\alpha|}, \quad|\alpha| \leq 10 d .
$$

Define $S_{\mathfrak{z}} \equiv S_{\mathfrak{z}}^{k}$ by

$$
S_{\mathfrak{z}}(y)=\int a_{k, \mathfrak{z}}(\xi) e^{i(\langle y, \xi\rangle-\varphi(\mathfrak{z}, \xi))} d \xi .
$$

Then for each $\mathcal{E} \subset \mathcal{Z}_{k}$ we have

$$
\operatorname{meas}\left(\left\{y \in \mathbb{R}^{d}:\left|\sum_{\mathfrak{z} \in \mathcal{E}} S_{\mathfrak{z}}\right|>\alpha\right\}\right) \leq C 2^{k\left(\frac{d+1}{2} p_{\ell}-1\right)} \alpha^{-p_{\ell}} \# \mathcal{E} \text {. }
$$

In the following subsections we prove some preparatory $L^{1}$ and $L^{2}$ estimates, then prove Proposition 2.2, and that Proposition 2.2 implies (6). In $\S 3$ we combine the dyadic estimates in Theorem 2.1.

2.1. $L^{1}$ estimates. $L^{1}$-estimates for the expressions $S_{\mathfrak{z}}$ can be found in [16]. In what follows we let $\Theta_{k}$ be a maximal $2^{-k / 2}$-separated set of unit vectors. Using a homogeneous extension of a partition of unity on the sphere one can split

$$
a_{k, \mathfrak{z}}(\xi)=\sum_{\theta \in \Theta_{k}} a_{k, \mathfrak{z}, \theta}(\xi)
$$

where $a_{k, \mathfrak{z}, \theta}$ is supported on the intersection of the cone $\left\{\xi:\left|\frac{\xi}{|\xi|}-\theta\right| \leq 2^{-k / 2}\right\}$ with the support of $a_{k, \mathfrak{z}}$; moreover if $u_{i}$ are unit vectors perpendicular to $\theta$ we have the estimates

$$
\left|\left\langle\theta, \nabla_{\xi}\right\rangle^{M_{1}} \prod_{i=1}^{M_{2}}\left\langle u_{i}, \nabla_{\xi}\right\rangle a_{k, \mathfrak{z}, \theta}(\xi)\right| \leq C\left(M_{1}, M_{2}\right) 2^{-k M_{1}} 2^{-k M_{2} / 2}
$$

whenever $M_{1}+M_{2} \leq 10 d$. Let

$$
S_{\mathfrak{z}, \theta}(y)=\int a_{k, \mathfrak{z}, \theta}(\xi) e^{i(\langle y, \xi\rangle-\varphi(\mathfrak{z}, \xi))} d \xi
$$

By homogeneity we have

$$
\phi_{\xi \xi}^{\prime \prime}(z, \theta) \theta=0 .
$$

Using this observation we get, as in [16], by an integration by parts

$$
\left|S_{\mathfrak{z}, \theta}(y)\right| \leq C_{d} 2^{k \frac{d+1}{2}}\left(1+2^{k}\left|\left\langle\varphi_{\xi}^{\prime}(\mathfrak{z}, \theta)-y, \theta\right\rangle\right|+2^{k / 2}\left|\Pi_{\theta^{\perp}}\left(\varphi_{\xi}^{\prime}(\mathfrak{z}, \theta)-y\right)\right|\right)^{-10 d} ;
$$

here $\Pi_{\theta} \perp$ denotes the projection to the orthogonal complement of $\theta$. This estimate implies $\left\|S_{\mathfrak{z}, \theta}\right\|_{1}=O(1)$ and therefore

$$
\left\|S_{\mathfrak{z}}\right\|_{1} \lesssim 2^{k \frac{d-1}{2}}
$$

Moreover we get for $1 \leq R \leq 2^{k}$,

$$
\int_{\left|\Pi_{\theta^{\perp}}\left(\varphi_{\xi}^{\prime}(\mathfrak{z}, \theta)-y\right)\right|}\left|S_{\mathfrak{z}, \theta}(y)\right| d y \lesssim \int_{\substack{\left.2^{-k} R\right)^{1 / 2} \\ w^{\prime} \in \mathbb{R}^{d-1} \mid \geq\left(2^{-k} R\right)^{1 / 2}}} \frac{2^{k(d-1) / 2}}{\left(1+2^{k / 2}\left|w^{\prime}\right|\right)^{10 d-2}} d w^{\prime} \lesssim R^{\frac{1-9 d}{2}},
$$


and similarly

$$
\int_{\substack{\left|\left\langle\varphi_{\xi}^{\prime}(\mathfrak{z}, \theta)-y, \theta\right\rangle\right| \\ \geq 2^{-k} R}}\left|S_{\mathfrak{z}, \theta}(y)\right| d y \lesssim \int_{\left|w_{d}\right| \geq 2^{-k} R} \frac{2^{k}}{\left(1+2^{k}\left|w_{d}\right|\right)^{9 d-1}} d w_{d} \lesssim R^{2-9 d} .
$$

Now clearly

$$
\left|\varphi_{\xi}^{\prime}(z, \theta)-\varphi_{\xi}^{\prime}(\widetilde{z}, \widetilde{\theta})\right| \lesssim|z-\widetilde{z}|+|\theta-\widetilde{\theta}|
$$

and by (11) also

Thus if

$$
\left|\left\langle\varphi_{\xi}^{\prime}(z, \theta)-\varphi_{\xi}^{\prime}(\widetilde{z}, \widetilde{\theta}), \theta\right\rangle\right| \lesssim|z-\widetilde{z}|+|\theta-\widetilde{\theta}|^{2}
$$

$$
V_{\theta}^{k}(z, R)=\left\{y:\left|\left\langle\varphi_{\xi}^{\prime}(z, \theta)-y, \theta\right\rangle\right| \leq R 2^{-k}, \quad\left|\Pi_{\theta^{\perp}}\left(\varphi_{\xi}^{\prime}(z, \theta)-y\right)\right| \leq\left(R 2^{-k}\right)^{1 / 2}\right\}
$$

then the above calculations give

$$
\left\|S_{\widetilde{\mathfrak{z}}, \widetilde{\theta}}\right\|_{L^{1}\left(\mathbb{R}^{d} \backslash V_{\theta(\mathfrak{z}, R)}^{k}\right)} \leq C\left(C_{1}\right) R^{-4 d} \quad \text { if }|\widetilde{\mathfrak{z}}-\mathfrak{z}| \leq C_{1} R 2^{-k}, \quad|\widetilde{\theta}-\theta| \leq C_{1}\left(R 2^{-k}\right)^{1 / 2}
$$

for $C_{1} \geq 1$.

2.2. Estimates for scalar products. Based on standard calculations for oscillatory integrals ([10], [18], [1], [11], [14]) we prove some estimates for scalar products $\left\langle S_{\mathfrak{z}}, S_{\mathfrak{z}^{\prime}}\right\rangle$; these results are closely related to the scalar product estimates in [6]. For the Fourier transforms we have

$$
\widehat{S}_{\mathfrak{z}}(\xi)=a_{k, \mathfrak{z}}(\xi) e^{-i \varphi(\mathfrak{z}, \xi)}
$$

and

$$
\begin{aligned}
(2 \pi)^{d}\left\langle S_{\mathfrak{z}}, S_{\widetilde{\mathfrak{z}}}\right\rangle & =\left\langle\widehat{S}_{\mathfrak{z}}, \widehat{S}_{\widetilde{\mathfrak{z}}}\right\rangle=\int a_{k, \mathfrak{z}}(\eta) \overline{a_{k, \widetilde{\mathfrak{z}}}(\eta)} e^{i(\varphi(\widetilde{\mathfrak{z}}, \eta)-\varphi(\mathfrak{z}, \eta))} d \eta \\
& =2^{k d} \int b_{k, \mathfrak{z}, \tilde{\mathfrak{z}}}(\xi) e^{i 2^{k}(\varphi(\widetilde{\mathfrak{z}}, \xi)-\varphi(\mathfrak{z}, \xi))} d \xi
\end{aligned}
$$

where $b_{k, \mathfrak{z}, \tilde{z}}$ is supported on a subset of diameter $O\left(\varepsilon^{2}\right)$ of the annulus $\{|\xi| \approx 1\}$, near $e_{1}$, with $\varepsilon$ sufficiently small. We may assume in what follows that $\mathfrak{z}, \widetilde{\mathfrak{z}}$ are in a neighborhood of the origin in $\mathbb{R}^{d+1}$, of diameter $\lesssim \varepsilon^{2}$. We split coordinates $z=\left(z^{\prime}, z_{d+1}\right)$, take advantage of (3) and get

$$
\left|\varphi_{\xi}^{\prime}(\mathfrak{z}, \xi)-\varphi_{\xi}^{\prime}(\widetilde{\mathfrak{z}}, \xi)\right| \geq c\left|\mathfrak{z}^{\prime}-\widetilde{\mathfrak{z}}^{\prime}\right|-C \varepsilon|\mathfrak{z} d+1-\widetilde{\mathfrak{z}} d+1|
$$

and after an integration by parts we get

$$
\left|\left\langle S_{\mathfrak{z}}, S_{\tilde{\mathfrak{z}}}\right\rangle\right| \lesssim \frac{2^{k d}}{\left(1+2^{k}|\mathfrak{z}-\widetilde{\mathfrak{z}}|\right)^{9 d}} \quad \text { if }\left|\mathfrak{z}^{\prime}-\widetilde{\mathfrak{z}}^{\prime}\right| \geq C_{1} \varepsilon|\mathfrak{z} d+1-\widetilde{\mathfrak{z}} d+1| .
$$

For $s \in[0,1]$ set $\mathfrak{z} s=\mathfrak{z}+s(\widetilde{\mathfrak{z}}-\mathfrak{z})$. If

$$
\left|\mathfrak{z}^{\prime}-\widetilde{\mathfrak{z}}^{\prime}\right| \leq C_{2} \varepsilon\left|\mathfrak{z}_{d+1}-\widetilde{\mathfrak{z}}_{d+1}\right|
$$

(with suitable $C_{1} \ll C_{2} \ll \varepsilon^{-1}$ ) we consider

$$
\frac{\varphi(\widetilde{\mathfrak{z}}, \xi)-\varphi(\mathfrak{z}, \xi)}{\widetilde{\mathfrak{z}} d+1-\mathfrak{z} d+1}=\int_{0}^{1}\left[\varphi_{z_{d+1}}^{\prime}\left(\mathfrak{z}_{s}, \xi\right)+\left\langle\frac{\widetilde{\mathfrak{z}}^{\prime}-\mathfrak{z}^{\prime}}{\widetilde{\mathfrak{z}} d+1-\mathfrak{z}_{d+1}}, \varphi_{z^{\prime}}^{\prime}\left(\mathfrak{z}_{s}, \xi\right)\right\rangle\right] d s .
$$

Note that $\frac{\varphi(\widetilde{z}, \xi)-\varphi(\mathfrak{z}, \xi)}{\tilde{\mathfrak{z}} d+1-\mathfrak{z} d+1}$ is a small perturbation of $\varphi_{z_{d+1}}^{\prime}(0, \xi)$ if $\varepsilon$ is sufficiently small. We apply the method of stationary phase (with parameters, [8]) in the $\xi^{\prime}$-variables, using (5). This yields

$$
\left|\left\langle S_{\mathfrak{z}}, S_{\mathfrak{z}}\right\rangle\right| \lesssim \frac{2^{k d}}{\left(1+2^{k}|\widetilde{\mathfrak{z}} d+1-\mathfrak{z} d+1|\right)^{\ell / 2}}, \quad \text { if }\left|\mathfrak{z}^{\prime}-\widetilde{\mathfrak{z}}\right| \leq C_{2} \varepsilon|\mathfrak{z} d+1-\widetilde{\mathfrak{z}} d+1|,
$$


and combining this with (17) we get

$$
\left|\left\langle S_{\mathfrak{z}}, S_{\mathfrak{z}}\right\rangle\right| \lesssim \frac{2^{k d}}{\left(1+2^{k}|\widetilde{\mathfrak{z}}-\mathfrak{z}|\right)^{\ell / 2}}
$$

whenever $|\mathfrak{z}-\widetilde{\mathfrak{z}}|=O\left(\varepsilon^{2}\right)$.

2.3. Proof of Proposition 2.2. If $\alpha \leq 2^{k \frac{d+1}{2}}$ then the desired inequality follows from (12). Indeed by Tshebyshev's inequality the left hand side of (9) is $\lesssim \alpha^{-1} 2^{k(d-1) / 2} \# \mathcal{E}$ which is dominated by the right hand side of (9) if $\alpha \leq 2^{k \frac{d+1}{2}}$.

In what follows we shall therefore assume that $\alpha>2^{k \frac{d+1}{2}}$ and set

$$
u_{k}(\alpha):=\left(\alpha 2^{-k \frac{d+1}{2}}\right)^{p_{\ell}}>1 .
$$

The argument is a variant of one in [6]; it is based on a Calderón-Zygmund type decomposition at height $u_{k}(\alpha)$ where volume is replaced by diameter.

By the usual Vitali procedure there is a finite (possibly empty) family $\mathfrak{B}^{k}$ of disjoint balls so that

$$
u_{k}(\alpha) 2^{k} \operatorname{diam}(B) \leq \#(\mathcal{E} \cap B) \quad \text { for } B \in \mathfrak{B}^{k} ;
$$

moreover if we remove the balls in $\mathfrak{B}^{k}$ and set

$$
\mathcal{E}_{*}=\mathcal{E} \backslash \bigcup_{B \in \mathfrak{B}^{k}} B
$$

then

$$
\#\left(\mathcal{E}_{*} \cap B\right) \leq C_{d} u_{k}(\alpha) 2^{k} \operatorname{diam}(B) \quad \text { for every ball } B \text {. }
$$

Since $\mathcal{E} \subset \mathcal{Z}_{k}$ which is $2^{-k}$-separated, we may assume that $\operatorname{diam}(B) \geq 2^{-k}$ if $B \in \mathfrak{B}^{k}$.

We need to establish the following two inequalities:

$$
\begin{aligned}
& \operatorname{meas}\left(\left\{y \in \mathbb{R}^{d}:\left|\sum_{B \in \mathfrak{B}^{k}} \sum_{\mathfrak{z} \in \mathcal{E} \cap B} S_{\mathfrak{z}}\right|>\alpha / 2\right\}\right) \leq C 2^{k\left(\frac{d+1}{2} p_{\ell}-1\right)} \alpha^{-p_{\ell}} \# \mathcal{E}, \\
& \operatorname{meas}\left(\left\{y \in \mathbb{R}^{d}:\left|\sum_{\mathfrak{z} \in \mathcal{E}_{*}} S_{\mathfrak{z}}\right|>\alpha / 2\right\}\right) \leq C 2^{k\left(\frac{d+1}{2} p_{\ell}-1\right)} \alpha^{-p_{\ell}} \# \mathcal{E} .
\end{aligned}
$$

Proof of (22). We first form an exceptional set as follows. Let $z_{B}$ denote the center of a ball $B \in \mathfrak{B}^{k}$ and let $R_{B}=10 d 2^{k} \operatorname{diam}(B) \gtrsim 1$. Let $\Theta(k, B)$ be a maximal $C_{1}\left(2^{-k} R_{B}\right)^{1 / 2}$ separated subset of $S^{d-1}$. Here $C_{1}$ is the constant in (14). Define (using the notation in $(13))$

$$
\mathcal{V}^{k}=\bigcup_{B \in \mathfrak{B}^{k}} \bigcup_{\vartheta \in \Theta(k, B)} V_{\vartheta}^{k}\left(z_{B}, R_{B}\right)
$$

Observe that meas $\left(V_{\vartheta}^{k}\left(z_{B}, R_{B}\right)\right)$ is $O\left(\left(R_{B} 2^{-k}\right)^{(d+1) / 2}\right)$ and $\# \Theta(k, B)=O\left(\left(2^{k} R_{B}^{-1}\right)^{(d-1) / 2}\right)$. Thus

$$
\begin{aligned}
\operatorname{meas}\left(\mathcal{V}^{k}\right) & \lesssim \sum_{B \in \mathfrak{B}^{k}} \sum_{\vartheta \in \Theta(k, B)} \operatorname{meas}\left(V_{\vartheta}^{k}\left(z_{B}, R_{B}\right)\right) \lesssim \sum_{B \in \mathfrak{B}^{k}} R_{B} 2^{-k} \\
& \lesssim \sum_{B \in \mathfrak{B}^{k}} \operatorname{diam}(B) \lesssim \sum_{B \in \mathfrak{B}^{k}} 2^{-k} \frac{\#(\mathcal{E} \cap B)}{u_{k}(\alpha)} \lesssim 2^{k\left(-1+\frac{d+1}{2} p_{\ell}\right)} \alpha^{-p_{\ell} \# \mathcal{E},}
\end{aligned}
$$

by the disjointness of the balls in $\mathfrak{B}^{k},(20)$, and the definition of $u_{k}(\alpha)$. 
To conclude the proof of $(22)$ we have to estimate the contribution in the complement of $\mathcal{V}^{k}$. For this we bound

$$
\operatorname{meas}\left(\left\{y \in \mathbb{R}^{d} \backslash \mathcal{V}^{k}:\left|\sum_{B \in \mathfrak{B}^{k}} \sum_{\mathfrak{z} \in \mathcal{E} \cap B} S_{\mathfrak{z}}\right|>\frac{\alpha}{2}\right\}\right) \lesssim \alpha^{-1}\left\|\sum_{B \in \mathfrak{B}^{k}} \sum_{\mathfrak{z} \in \mathcal{E} \cap B} S_{\mathfrak{z}}\right\|_{L^{1}\left(\mathbb{R}^{d} \backslash \mathcal{V}^{k}\right)} .
$$

Now fix $B$. For every $\theta \in \Theta_{k}$ we may choose a $\vartheta=\vartheta_{B}(\theta) \in \Theta(k, B)$ so that $\left|\vartheta_{B}(\theta)-\theta\right| \leq$ $C_{1}\left(R_{B} 2^{-k}\right)^{-1 / 2}$. Recalling $S_{\mathfrak{z}}=\sum_{\theta \in \Theta_{k}} S_{\mathfrak{z}, \theta}$, we see

$$
\begin{aligned}
\| & \sum_{B \in \mathfrak{B}^{k}} \sum_{\mathfrak{z} \in \mathcal{E} \cap B} S_{\mathfrak{z}} \|_{L^{1}\left(\mathbb{R}^{d} \backslash \mathcal{V}^{k}\right)} \\
\leq & \sum_{B \in \mathfrak{B}^{k}} \sum_{\mathfrak{z} \in \mathcal{E} \cap B} \sum_{\theta \in \Theta_{k}}\left\|S_{\mathfrak{z}, \theta}\right\|_{L^{1}\left(\mathbb{R}^{d} \backslash V_{\vartheta_{B}(\theta)}^{k}\left(z_{B}, R_{B}\right)\right)} \\
& \lesssim \sum_{B \in \mathfrak{B}^{k}} \sum_{\mathfrak{z} \in \mathcal{E} \cap B} \sum_{\theta \in \Theta_{k}} R_{B}^{-4 d} \lesssim \sum_{B \in \mathfrak{B}^{k}} \sum_{\mathfrak{z} \in \mathcal{E} \cap B} 2^{k(d-1) / 2} R_{B}^{-4 d} .
\end{aligned}
$$

For the second inequality we use (14) and the last one follows from $\# \Theta_{k}=O\left(2^{k(d-1) / 2}\right)$. Now we note that $2^{k \frac{d-1}{2}} \alpha^{-1}=2^{k\left(-1+\frac{d+1}{2} p_{\ell}\right)} \alpha^{-p_{\ell}} u_{k}(\alpha)^{1-\frac{1}{p_{\ell}}}$. Thus

$$
\begin{aligned}
& \operatorname{meas}\left(\left\{y \in \mathbb{R}^{d} \backslash \mathcal{V}^{k}:\left|\sum_{B \in \mathfrak{B}^{k}} \sum_{\mathfrak{z} \in \mathcal{E} \cap B} S_{\mathfrak{z}}\right|>\frac{\alpha}{2}\right\}\right) \lesssim \alpha^{-1} \sum_{B \in \mathfrak{B}^{k}} \sum_{\mathfrak{z} \in \mathcal{E} \cap B} 2^{k(d-1) / 2} R_{B}^{-4 d} \\
& \lesssim 2^{k\left(-1+\frac{d+1}{2} p_{\ell}\right)} \alpha^{-p_{\ell}} u_{k}(\alpha)^{1-\frac{1}{p_{\ell}}} \sum_{B \in \mathfrak{B}^{k}} \sum_{\mathfrak{z} \in \mathcal{E} \cap B} R_{B}^{-4 d} .
\end{aligned}
$$

By (20) we have for $B \in \mathfrak{B}^{k}$

$$
u_{k}(\alpha) \lesssim \frac{\#(\mathcal{E} \cap B)}{R_{B}} \lesssim R_{B}^{d}
$$

and therefore

$$
\begin{aligned}
& \operatorname{meas}\left(\left\{y \in \mathbb{R}^{d} \backslash \mathcal{V}^{k}:\left|\sum_{B \in \mathfrak{B}^{k}} \sum_{\mathfrak{z} \in \mathcal{E} \cap B} S_{\mathfrak{z}}\right|>\frac{\alpha}{2}\right\}\right) \\
& \lesssim 2^{k\left(-1+\frac{d+1}{2} p_{\ell}\right)} \alpha^{-p_{\ell}} u_{k}(\alpha)^{1-\frac{1}{p_{\ell}}-4} \sum_{B \in \mathfrak{B}^{k}} \#(\mathcal{E} \cap B) \lesssim 2^{k\left(-1+\frac{d+1}{2} p_{\ell}\right)} \alpha^{-p_{\ell}} \# \mathcal{E}
\end{aligned}
$$

since $u_{k}(\alpha) \geq 1$ and $R_{B} \gtrsim 1$.

Proof of (23). We check from (19) that

$$
2^{k d} u_{k}(\alpha)^{2 / \ell} \alpha^{-2}=2^{k\left(-1+\frac{d+1}{2} p_{\ell}\right)} \alpha^{-p_{\ell}} .
$$

Thus by Tshebyshev's inequality it suffices to prove

$$
\left\|\sum_{\mathfrak{z} \in \mathcal{E}_{*}} S_{\mathfrak{z}}\right\|_{2}^{2} \lesssim 2^{k d} u_{k}(\alpha)^{2 / \ell} \# \mathcal{E}_{*}
$$


We set

$$
\begin{aligned}
L & :=u_{k}(\alpha)^{2 / \ell}, \\
I(n, L) & :=\left[n 2^{-k} L,(n+1) 2^{-k} L\right), \\
\mathcal{E}(n, L) & :=\left\{\mathfrak{z} \in \mathcal{E}_{*}: \mathfrak{z} d+1 \in I(n, L)\right\}, \\
\mathfrak{S}_{n} & :=\sum_{\mathfrak{z} \in \mathcal{E}(n, L)} S_{\mathfrak{z}} .
\end{aligned}
$$

Now

$$
\left\|\sum_{\mathfrak{z} \in \mathcal{E}_{*}} S_{\mathfrak{z}}\right\|_{2}^{2} \lesssim \sum_{n} \sum_{\tilde{n}:|n-\tilde{n}| \leq 4}\left\langle\mathfrak{S}_{n}, \mathfrak{S}_{\tilde{n}}\right\rangle+\sum_{n} \sum_{\tilde{n}:|n-\tilde{n}|>4}\left\langle\mathfrak{S}_{n}, \mathfrak{S}_{\tilde{n}}\right\rangle=: I+I I .
$$

For $I$ we use the Schwarz inequality and then (17) to get

$$
\begin{aligned}
& |I| \lesssim \sum_{n}\left\|\mathfrak{S}_{n}\right\|_{2}^{2}=\sum_{n}\left\|\sum_{\mathfrak{z} d+1 \in I(n, L)} \sum_{\mathfrak{z}^{\prime}} S_{\left(\mathfrak{z}^{\prime}, \mathfrak{d} d+1\right)}\right\|_{2}^{2} \\
& \lesssim L \sum_{n} \sum_{\mathfrak{z} d+1 \in I(n, L)}\left\|\sum_{\substack{\mathfrak{z}^{\prime}: \\
\left(\mathfrak{z}^{\prime}, \mathfrak{z} d+1\right) \in \mathcal{E}_{*}}} S_{\left(\mathfrak{z}^{\prime}, \mathfrak{z} d+1\right)}\right\|_{2}^{2} \\
& \lesssim L \sum_{n} \sum_{\mathfrak{z} d+1 \in I(n, L)} \sum_{\substack{\mathfrak{z}^{\prime}: \\
\left(\mathfrak{z}^{\prime}, \mathfrak{d} d+1\right) \in \mathcal{E}}} \sum_{\widetilde{\mathfrak{z}}^{\prime}} 2^{k d}\left(1+2^{k}\left|\mathfrak{z}^{\prime}-\widetilde{\mathfrak{z}}^{\prime}\right|\right)^{-8 d} \lesssim L 2^{k d} \# \mathcal{E} .
\end{aligned}
$$

For $I I$ we use (18) and estimate

$$
|I I| \lesssim \sum_{\mathfrak{z} \in \mathcal{E}_{*}} \sum_{\substack{\tilde{\mathfrak{z}} \in \mathcal{E}_{*} \\ \mid \mathfrak{z} d+1}}\left|\left\langle S_{\mathfrak{z}}, S_{\mathfrak{z}}\right\rangle\right| \lesssim \sum_{\mathfrak{z} \in \mathcal{E}_{*} \mid \geq 2^{-k} L} \sum_{\substack{\mathfrak{\mathfrak { z }} \in \mathcal{E}_{*}: \\ \mid \mathfrak{z} d+1-\mathfrak{z} \mathfrak{z} d+1}} \frac{2^{k d}}{\left(1+2^{k}|\mathfrak{z}-\widetilde{\mathfrak{z}}|\right)^{\ell / 2} L}
$$

By (21) we have for $R \geq 1$ and fixed $\mathfrak{z}$

$$
\sum_{\substack{\tilde{\mathfrak{z}} \in \mathcal{E}_{*} \\ 2^{-k} \leq|\mathfrak{z}-\widetilde{\mathfrak{z}}| \leq 2^{1-k} R}}\left(1+2^{k}|\mathfrak{z}-\widetilde{\mathfrak{z}}|\right)^{-\ell / 2} \lesssim u_{k}(\alpha) R^{1-\frac{\ell}{2}}
$$

and since $\ell / 2>1$ we get (after setting $R=2^{m}$ and summing over $m$ with $2^{m} \geq L$ )

$$
|I I| \lesssim 2^{k d} u_{k}(\alpha) L^{1-\frac{\ell}{2}} \# \mathcal{E} .
$$

Hence

$$
I+I I \lesssim 2^{k d} \# \mathcal{E}\left(L+u_{k}(\alpha) L^{1-\frac{\ell}{2}}\right)
$$

and with the optimal choice of $L=\left[u_{k}(\alpha)\right]^{2 / \ell}$ we obtain (25).

2.4. Proof that Proposition 2.2 implies (6). We shall first assume that in (2)

$$
\chi_{k}\left(z, y, 2^{-k} \xi\right)=\eta_{k}\left(z, 2^{-k} \xi\right) \chi_{\circ}(y)
$$

where $\chi_{\circ} \in C_{c}^{\infty}\left(\mathbb{R}^{d}\right)$ is supported on a small neighborhood of the origin but so that $\chi_{\circ}(y)=$ 1 for $y \in E$; moreover $\eta_{k}$ is compactly supported in a set of diameter $O\left(\varepsilon^{2}\right)$ near $(z, \xi)=$ $\left(0, e_{1}\right)$, and the derivatives of $\eta_{k}$ up to order $10 d$ are uniformly bounded. 
Let $Q_{\mathfrak{z}}=\prod_{i=1}^{d+1}\left[\mathfrak{z}_{i}, \mathfrak{z}_{i}+2^{-k}\right]$. For $m \geq 0$ let

$$
\begin{aligned}
\mathcal{E}_{m} & =\left\{\mathfrak{z} \in \mathcal{Z}_{k}: 2^{-k(d+1)-m-1}<\left|Q_{\mathfrak{z}} \cap E\right| \leq 2^{-k(d+1)-m}\right\}, \\
E_{m} & =\bigcup_{\mathfrak{z} \in \mathcal{E}_{m}} Q_{\mathfrak{z}} \cap E .
\end{aligned}
$$

And we also set

$$
\begin{aligned}
a_{k, \mathfrak{z}, m}(\xi) & =2^{m+(k+1) d} \int_{Q_{\mathfrak{z}} \cap E_{m}} \eta_{k}\left(z, 2^{-k} \xi\right) e^{i(\varphi(\mathfrak{z}, \xi)-\varphi(z, \xi))} d z, \\
S_{\mathfrak{z}, m}(y) & =\int a_{k, \mathfrak{z}, m}(\xi) e^{i(\langle y, \xi\rangle-\varphi(\mathfrak{z}, \xi))} d \xi
\end{aligned}
$$

Then it follows that

$$
T_{k}^{*} \chi_{E}(y)=\sum_{m=0}^{\infty} 2^{-m-(k+1) d} S_{\mathfrak{z}, m}(y) .
$$

Since $\partial_{\xi}^{\alpha}(\varphi(\mathfrak{z}, \xi)-\varphi(z, \xi))=O\left(2^{-k}\right)$ for any multiindex $\alpha$ it is easy to see that $a_{\cdot, \cdot, m}$ satisfies (7) uniformly in $m$. Hence, the result of Proposition 2.2 can be applied to $\sum_{\mathfrak{z} \in \mathcal{E}_{m}} S_{\mathfrak{z}, m}(y)$ and we get uniform bounds. Thus

$$
\left\|\sum_{\mathfrak{z} \in \mathcal{E}_{m}} S_{\mathfrak{z}, m}\right\|_{L^{p_{\ell}, \infty}} \lesssim 2^{k\left(\frac{d+1}{2}-\frac{1}{p_{\ell}}\right)}\left(\# \mathcal{E}_{m}\right)^{1 / p_{\ell}} \lesssim 2^{k\left(\frac{d+1}{2}-\frac{1}{p_{\ell}}\right)} 2^{m / p_{\ell}}\left(2^{k(d+1)} \operatorname{meas}\left(E_{m}\right)\right)^{1 / p_{\ell}}
$$

with implicit constants independent of $m$. For the second inequality we use

$$
\# \mathcal{E}_{m} \lesssim 2^{m} 2^{k(d+1)} \operatorname{meas}\left(E_{m}\right)
$$

which follows from (27). Consequently we get

$$
\begin{aligned}
& \left\|T_{k}^{*} \chi_{E}\right\|_{L^{p_{\ell}, \infty}} \lesssim \sum_{m=0}^{\infty} 2^{-m-(k+1) d}\left\|S_{\mathfrak{z}, m}\right\|_{L^{p_{\ell}, \infty}} \\
& \lesssim \sum_{m=0}^{\infty} 2^{-m\left(1-\frac{1}{p_{\ell}}\right)} 2^{k\left(\frac{d}{p_{\ell}}-\frac{d+1}{2}\right)}\left(\operatorname{meas}\left(E_{m}\right)\right)^{1 / p_{\ell}} \lesssim 2^{k\left(\frac{d}{p_{\ell}}-\frac{d+1}{2}\right)}(\operatorname{meas}(E))^{1 / p_{\ell}}
\end{aligned}
$$

which is the desired estimate.

Finally we have to remove the assumption (26). Here one uses Fourier series in $y$ and expands $\chi_{k}\left(z, y, 2^{-k} \xi\right)=\sum_{\nu \in \mathbb{Z}^{d}} c_{k, \nu} \eta_{k, \nu}\left(z, 2^{-k} \xi\right) e^{i\langle y, \nu\rangle}$ where the functions $\eta_{k, \nu}\left(z, 2^{-k} \xi\right)$ are as before but now with a bound that decays fast in $\nu$. We note that multiplication with $e^{i\langle y, \nu\rangle}$ does not affect the $L^{p_{\ell}, 1}$ norm, apply the previous bounds to the summands and sum in $\nu$ using the rapid decay in $\nu$.

\section{Combining the FREQuency LOCAlized Pieces}

We now combine the previous estimates on the operators $T_{k}$ and prove the following result in Triebel-Lizorkin spaces $F_{a, s}^{q}$. Recall ([19]) that if $\left\{L_{k}\right\}_{k=0}^{\infty}$ is a standard inhomogeneous dyadic frequency decomposition then the norm $\|f\|_{F_{a, s}^{q}}$ can be defined as the $L^{q}\left(\ell^{s}\right)$ norm of the sequence $\left\{2^{k a} L_{k} f\right\}$. In view of the embeddings $L^{q}=F_{0,2}^{q} \subset F_{0, q}^{q}$ for $q \geq 2, L^{q} \supset F_{0, r}^{q}$ for $r \leq 2$ the following result sharpens Theorem 1.1. For the case $r \geq 1$ one could argue by duality and follow [6] but we shall rely on a result in [15] which gives an estimate for all $r>0$. 
Theorem 3.1. Let $\ell \geq 3$, let $z, y$ be coordinate patches in $\mathbb{R}^{d+1}, \mathbb{R}^{d}$, resp., let $\mathcal{F} \in$ $I^{\mu-1 / 4}(Z, y ; \mathcal{C})$, with Schwartz kernel compactly supported in $\mathcal{Z} \times \mathcal{y}$, and let $\mathcal{C}$ satisfy hypothesis $\mathcal{H}(\ell)$. Suppose $\frac{2 \ell}{\ell-2}<q<\infty, a=\mu+b+d(1 / 2-1 / q)-1 / 2$ and $r>0$. Then $\mathcal{F}$ maps $F_{a, q}^{q}\left(\mathbb{R}^{d}\right)$ boundedly to $F_{b, r}^{q}\left(\mathbb{R}^{d+1}\right)$.

We state (a slight variant of) the result from [15]. In this setting one is given operators $\mathcal{T}_{k}$ defined on the Schwartz space $\mathcal{S}\left(\mathbb{R}^{d_{1}}\right)$,

$$
\mathcal{T}_{k} f(z)=\int \mathcal{K}_{k}(z, y) f(y) d y, \quad z \in \mathbb{R}^{d_{2}}
$$

and each $\mathcal{K}_{k}$ is continuous and bounded. Let $\zeta \in \mathcal{S}\left(\mathbb{R}^{d_{2}}\right)$ and $\zeta_{k}(z)=2^{k d_{2}} \zeta\left(2^{k} z\right)$, and define $P_{k} g=\zeta_{k} * g$.

Theorem $3.2([15])$. Let $d_{1} \leq d_{2}, 0<\gamma<d_{2}, \varepsilon>0,1<q_{0}<q<\infty$, and assume

$$
\begin{aligned}
& \sup _{k>0} 2^{k \gamma / q_{0}}\left\|\mathcal{T}_{k}\right\|_{L^{q_{0}}\left(\mathbb{R}^{d_{1}}\right) \rightarrow L^{q_{0}}\left(\mathbb{R}^{d_{2}}\right)}<\infty, \\
& \sup _{k>0}\left\|\mathcal{T}_{k}\right\|_{L^{\infty}\left(\mathbb{R}^{d_{1}}\right) \rightarrow L^{\infty}\left(\mathbb{R}^{d_{2}}\right)}<\infty .
\end{aligned}
$$

Furthermore assume that for each cube $Q$ there is a measurable set $\mathcal{W}_{Q} \subset \mathbb{R}^{d_{2}}$ so that

$$
\operatorname{meas}\left(\mathcal{W}_{Q}\right) \leq C \max \left\{|Q|^{1-\gamma / d_{2}},|Q|\right\}
$$

and there is $\delta>0$ such that for every $k \in \mathbb{N}$ and every cube $Q$ with $2^{k} \operatorname{diam}(Q) \geq 1$

$$
\sup _{x \in Q} \int_{\mathbb{R}^{d_{2}} \backslash \mathcal{W}_{Q}}\left|\mathcal{K}_{k}(x, y)\right| d y \leq C \max \left\{\left(2^{k} \operatorname{diam}(Q)\right)^{-\delta}, 2^{-k \delta}\right\} .
$$

Then for $q_{0}<q<\infty, r>0$

$$
\left\|\left(\sum_{k} 2^{k \gamma r / q}\left|P_{k} \mathcal{T}_{k} f_{k}\right|^{r}\right)^{1 / r}\right\|_{q} \lesssim\left\|\left(\sum_{k}\left\|f_{k}\right\|_{q}^{q}\right)^{1 / q}\right\|_{q}
$$

This (or a slightly sharper version) was formulated in [15] only for the case $d_{1}=d_{2}$, but the result there implies the version cited above. Indeed if $d_{1}<d_{2}$ we can define an operator $\widetilde{\mathcal{T}}_{k}$ on functions $F$ on $\mathbb{R}^{d_{1}} \times \mathbb{R}^{d_{2}-d_{1}}$ by

$$
\widetilde{\mathcal{T}}_{k} F(z)=\iint K_{k}(z, y) \chi(w) F(y, w) d y d w
$$

where $\chi$ is a nontrivial $C_{c}^{\infty}\left(\mathbb{R}^{d_{2}-d_{1}}\right)$ function. The assumptions on $\mathcal{T}_{k}$ imply the corresponding assumptions on $\widetilde{\mathcal{T}}_{k}$, by Minkowski's and Hölder's inequalities. Thus the equidimensional case in [15] may be applied and if in the conclusion we specialize to tensor products, $F(y, w)=f(y) \chi_{1}(w)$ we get the above generalization.

In order to prepare for our application of Theorem 3.2 we let $T_{k} f(z)=\int K_{k}(z, y) f(y) d y$ with $K_{k}$ as in (2). Let $\beta_{0}$ be a $C^{\infty}$-function supported in $\left\{\eta \in \mathbb{R}^{d+1}:|\eta| \leq 3 / 2\right\}$ so that $\beta_{0}(\eta)=1$ for $|\eta| \leq 1$; and, for $k \geq 1$ let $\beta_{k}(\eta)=\beta_{0}\left(2^{-k}(\eta)\right)-\beta_{0}\left(2^{1-k}(\eta)\right)$. Define $L_{k}$ on functions on $\mathbb{R}^{d+1}$ by $\widehat{L_{k} g}=\beta_{k}(\eta) \widehat{g}(\eta)$. We use calculations in [8] and first observe that there is a constant $A_{0}>1$ so that

$$
\left\|L_{\widetilde{k}} T_{k}\right\|_{L^{q} \rightarrow L^{q}} \leq C_{N} \min \left\{2^{-k N}, 2^{-\widetilde{k} N}\right\} \quad \text { if }|k-\widetilde{k}| \geq A_{0} .
$$


This follows from the assumption that $\mathcal{C}$ does not meet the zero sections, which, by homogeneity implies that $c_{1}|\xi| \leq\left|\varphi_{z}^{\prime}(z, \xi)\right| \leq C_{1}|\xi|$. The kernel $\mathcal{K}_{k \widetilde{k}}$ of $L_{\widetilde{k}} T_{k}$ is given by

$$
\left.\mathcal{K}_{k \widetilde{k}}(z, y)=\iiint \beta_{\widetilde{k}} \eta\right) \chi_{k}\left(z, y, 2^{-k} \xi\right) e^{i(\langle z-w, \eta\rangle+\varphi(w, \xi)-\langle y, \xi\rangle)} d w d \eta d \xi
$$

and if $k-\widetilde{k}$ are sufficiently large then $\left|\eta+\varphi_{w}^{\prime}(w, \xi)\right| \approx \max \{|\xi|,|\eta|\}$ on the support of the amplitude. Thus in this case we may use an integration by parts in $w$ (followed by an integration by parts in $\xi$ when $z$ is large) to show that the kernels $\mathcal{K}_{k \widetilde{k}}$ of $L_{\widetilde{k}} T_{k}$ satisfy the estimate

$$
\left|\mathcal{K}_{k \widetilde{k}}(z, y)\right| \leq C_{N} 2^{-k N}(1+|z|)^{-N}
$$

and vanish for $y$ in the complement of a fixed compact set. Thus (32) follows. Similarly if $\left\{\mathcal{L}_{k}\right\}_{k=0}^{\infty}$ is the corresponding frequency decomposition in $\mathbb{R}^{d}$ we also see that $T_{k} \mathcal{L}_{\widetilde{k}}$ has $L^{q}\left(\mathbb{R}^{d}\right) \rightarrow L^{q}\left(\mathbb{R}^{d+1}\right)$ operator norm $\leq C_{N} \min \left\{2^{-k N}, 2^{-\widetilde{k} N}\right\}$ if $|k-\widetilde{k}|>4$.

From these preliminary remarks it follows quickly that for the proof of Theorem 3.1 it suffices to prove the inequalities

$$
\left\|\left(\sum_{k} 2^{k b r}\left|2^{k \mu} L_{k+i_{1}} T_{k} \mathcal{L}_{k+i_{2}} g\right|^{r}\right)^{1 / r}\right\|_{q} \lesssim\left\|\left(\sum_{k} 2^{k a q}\left\|\mathcal{L}_{k+i_{2}} g\right\|_{q}^{q}\right)^{1 / q}\right\|_{q},\left|i_{1}\right| \leq A_{0}, \quad\left|i_{2}\right| \leq 4
$$

with $a=\mu+b+d(1 / 2-1 / q)-1 / 2$. Setting $\mathcal{T}_{k}=2^{-k \frac{d-1}{2}} T_{k}$ and $f_{k}=2^{k a} \mathcal{L}_{k+i_{2}} g$, the preceding inequality follows from

$$
\left\|\left(\sum_{k} 2^{k \frac{d}{q} r}\left|L_{k+i_{1}} \mathcal{T}_{k} f_{k}\right|^{r}\right)^{1 / r}\right\|_{q} \lesssim\left\|\left(\sum_{k}\left\|f_{k}\right\|_{q}^{q}\right)^{1 / q}\right\|_{q}, \quad\left|i_{1}\right| \leq A_{0}
$$

for $q_{0}<q<\infty$, where $q_{0}>\frac{2 \ell}{\ell-2}$. This in turn follows from an application of Theorem 3.2 with $d_{1}=d, d_{2}=d+1, \gamma=d$, and $P_{k}=L_{k+i_{1}}$. The hypothesis (29) follows from the calculations in [16] ( $c f$. also $\S 2.1$ above). The hypothesis (28) for $\frac{2 \ell}{\ell-2}<q<\infty$ follows from Theorem 2.1 and (29) by interpolation.

If $\operatorname{diam}(Q)>\varepsilon$ then $\mathcal{W}_{Q}$ is simply an expanded cube and (31) follows by the support assumption of $K_{k}$. If $\operatorname{diam}(Q)<\varepsilon$ the exceptional sets are formed as in $\S 2.1$. For $\theta \in S^{d-1}$ we set

$$
W_{\theta}\left(z_{Q}, C\right)=\left\{y:\left|\left\langle\varphi_{\xi}^{\prime}\left(z_{Q}, \theta\right)-y, \theta\right\rangle\right| \leq C \operatorname{diam}(Q),\left|\Pi_{\theta^{\perp}}\left(\varphi_{\xi}^{\prime}(z, \theta)-y\right)\right| \leq C(\operatorname{diam}(Q))^{1 / 2}\right\}
$$

and if $\Theta_{Q}$ is a maximal set of $(\operatorname{diam}(Q))^{1 / 2}$ separated unit vectors we set

$$
\mathcal{W}_{Q}=\bigcup_{\theta \in \Theta_{Q}} W_{\theta}\left(z_{Q}, C\right)
$$

Then the measure of $\mathcal{W}_{Q}$ is $O(\operatorname{diam}(Q))=O\left(|Q|^{1-\frac{d}{d+1}}\right)$ so that (30) holds. The hypothesis (31) (even with large $\delta$ ) holds by the calculations in $\S 2.1$ (cf. (14)).

\section{Remarks on the constant coefficient Case}

We now let $\rho$ be a $C^{\infty}\left(\mathbb{R}^{d} \backslash\{0\}\right)$ function which is homogeneous of degree 1 , so that $\rho(\xi) \neq 0$ for $\xi \neq 0$. We are interested in space time estimates for $U_{t} \equiv e^{-i t \rho(D)}$ defined by

$$
\widehat{U_{t} f}(\xi)=e^{i t \rho(\xi)} \widehat{f}(\xi)
$$

and obtain a result under a decay assumption for the Fourier transform of surface carried measure on

$$
\Sigma_{\rho}=\{\xi: \rho(\xi)=1\}
$$


Theorem 4.1. Let $\kappa>1$ and let $\rho$ be as above such that the surface measure d $\sigma$ of $\Sigma_{\rho}$ satisfies

$$
\sup _{\xi}(1+|\xi|)^{\kappa}|\widehat{d \sigma}(\xi)|<\infty .
$$

Let $\frac{2 \kappa}{\kappa-1}<q<\infty$ and let $I$ be a compact time interval. There is $C>0$ such that

$$
\left(\int_{I}\left\|e^{i t \rho(D)} f\right\|_{L^{q}\left(\mathbb{R}^{d}\right)}^{q} d t\right)^{1 / q} \leq C\|f\|_{B_{\alpha, q}^{q}\left(\mathbb{R}^{d}\right)}, \quad \alpha=\frac{d-1}{2}-\frac{d}{q}
$$

for all $f \in B_{\alpha, q}^{q}\left(\mathbb{R}^{d}\right)$.

Remark. Under the assumption that $\Sigma_{\rho}$ has nonvanishing curvature everywhere this follows from Theorem 3.1 above. We note that in this particular case a weaker result with $\alpha>(d-1) / 2-d / q$ is already in [7].

Sketch of proof. We will assume that $I=[-1,1]$, as one can use rescaling to reduce to this case. Fix $k$ and define $S_{x, t} \equiv S_{x, t}^{k}$ by

$$
S_{x, t}(y)=\int e^{i\langle x-y, \xi\rangle+i t \rho(\xi)} \chi\left(2^{-k} \xi\right) d \xi
$$

and as before we may assume that the support of $\chi$ has diameter $\leq \varepsilon^{2}$, for sufficently small $\varepsilon>0$, and is contained in $\{1 / 2<|\xi| \leq 2\}$. Fix $\xi_{\circ} \in \Sigma_{\rho}$ and $\rho_{\circ}>0$ so that $\rho_{\circ} \xi_{\circ} \in \operatorname{supp}(\chi)$. Let $u \mapsto \Xi(u)$ be a parametrization of $\Sigma_{\rho}$ near $\xi_{\circ}$ with parameter $u \in \mathbb{R}^{d-1}$ near $u_{\circ}$, and $\Xi\left(u_{\circ}\right)=\xi_{\circ}$. Let $\mathfrak{n}_{\circ}$ be the outer normal unit vector to $\Sigma_{\rho}$ at $\xi_{\circ}$. Let $\Gamma$ be the cone formed by the $(\rho \Xi(u), \rho)$ with $\rho>0$ and $u$ near $u_{\circ}$. Let $\vec{N}_{\circ}=\mathfrak{n}_{\circ}-\left\langle\xi_{\circ}, \mathfrak{n}_{\circ}\right\rangle \vec{e}_{d+1}$, which is a normal vector to $\Gamma$ at $\left(\rho \Xi\left(u_{\circ}\right), \rho\right)$. By finite decompositions of $\chi$ we may further assume that

$$
S_{x, t}(y)=\int \beta\left(2^{-k} \xi\right) e^{i(\langle x-y, \xi\rangle+i t \rho(\xi))} d \xi
$$

where $\beta \in C_{c}^{\infty}$ supported in an $\varepsilon^{2}$-neighborhood of $\rho_{\circ} \Xi\left(u_{\circ}\right)$.

The proof of Theorem 4.1 is a straightforward variant of the proof of Theorem 3.1 once we have established the two appropriate replacements for the scalar product bounds (18) and (17), namely

$$
\left|\left\langle S_{x, t}, S_{\tilde{x}, \tilde{t}}\right\rangle\right| \lesssim 2^{k d}\left(1+2^{k}|x-\tilde{x}|+2^{k}|t-\tilde{t}|\right)^{-\kappa},
$$

and a better estimate when $\left(x-\tilde{x}, t-\tilde{t}\right.$ ) is orthogonal (or near orthogonal) to $\vec{N}_{\circ}$ :

$$
\begin{aligned}
\left|\left\langle S_{x, t}, S_{\tilde{x}, \tilde{t}}\right\rangle\right| \leq C_{M} 2^{k d}\left(1+2^{k} \mid x-\right. & \left.\tilde{x}\left|+2^{k}\right| t-\tilde{t} \mid\right)^{-M} \\
& \text { if }\left|\left\langle x-\tilde{x}, \mathfrak{n}_{\circ}\right\rangle-(t-\tilde{t})\left\langle\xi_{\circ}, \mathfrak{n}_{\circ}\right\rangle\right| \leq \epsilon_{\circ}|(x-\tilde{x}, t-\tilde{t})|
\end{aligned}
$$

for a small $\epsilon_{\circ}>0$.

As before $(2 \pi)^{d}\left\langle S_{x, t}, S_{\tilde{x}, \tilde{t}}\right\rangle=\left\langle\widehat{S}_{x, t}, \widehat{S}_{\tilde{x}, \tilde{t}}\right\rangle$. We scale and then use generalized polar coordinates $\xi=\rho \Xi(u)$ to write

$$
\left\langle\widehat{S}_{x, t}, \widehat{S}_{\tilde{x}, \tilde{t}}\right\rangle=2^{k d} \iint b(\rho, u) e^{i 2^{k} \rho(\langle x-\tilde{x}, \Xi(u)\rangle+t-\tilde{t})} d u d \rho
$$

where $b$ is smooth and supported near $\left(\rho_{\circ}, u_{\circ}\right)$. Now for any $\chi \in C_{c}^{\infty}$ we have $|\widehat{\chi d \sigma}(\xi)| \lesssim$ $(1+|\xi|)^{\kappa}$, by assumption (34). We apply this to the inner integral of (37) and obtain

$$
\left|\left\langle S_{x, t}, S_{\tilde{x}, \tilde{t}}\right\rangle\right| \lesssim 2^{k d}\left(1+2^{k}|x-\tilde{x}|\right)^{-\kappa}
$$


Let $B=2 \max \left\{|\xi|: \xi \in \Sigma_{\rho}\right\}$. By an integration by parts in $\rho$ (after interchanging the order of integration in (37)) we obtain

$$
\left|\left\langle S_{x, t}, S_{\tilde{x}, \tilde{t}}\right\rangle\right| \leq C_{N} 2^{k d}\left(1+2^{k}|t-\tilde{t}|\right)^{-N} \quad \text { if }|t-\tilde{t}| \geq B|x-\tilde{x}|,
$$

and (35) follows from (38) and (39).

We now prove (36) and in view of (39) we may assume $|t-\tilde{t}|<B|x-\tilde{x}|$. We distinguish two cases. In the first case we assume $\left|\left\langle\frac{x-\tilde{x}}{|x-\tilde{x}|}, \mathfrak{n}_{\circ}\right\rangle\right| \leq 1-\varepsilon$. We note that $\left|\Xi(u)-\Xi\left(u_{\circ}\right)\right|=$ $O\left(\varepsilon^{2}\right)$ and $\left|\left(\nabla_{u}\langle x-\tilde{x}, \Xi(u)\rangle\right)_{u=u_{o}}\right| \sim\left|\Pi_{\mathfrak{n}^{\perp}}(x-\tilde{x})\right|$. Hence we have $\left|\nabla_{u}\langle x-\tilde{x}, \Xi(u)\rangle\right| \geq$ $c \varepsilon|x-\tilde{x}|$ on the support of $b$ provided that $\varepsilon$ is sufficiently small, and higher derivatives of $\langle x-\tilde{x}, \Xi(u)\rangle$ are $O(|x-\tilde{x}|)$. Thus, integrating by parts in the inner $u$-integral in (37), we get (36). We now consider the second case $\left|\left\langle\frac{x-\tilde{x}}{|x-\tilde{x}|}, \mathfrak{n}_{\circ}\right\rangle\right| \geq 1-\varepsilon$. This means that $\frac{x-\tilde{x}}{|x-\tilde{x}|}=s \mathfrak{n}_{\circ}+O(\varepsilon)$ where $s=1$ or $s=-1$. From the condition on $(x-\tilde{x}, t-\tilde{t})$ in (36) we see that the $\rho$-derivative of the phase is

$$
\begin{aligned}
\langle x-\tilde{x}, \Xi(u)\rangle+t-\tilde{t} & =\left\langle x-\tilde{x}, \xi_{\circ}\right\rangle+\frac{\left\langle x-\tilde{x}, \mathfrak{n}_{\circ}\right\rangle}{\left\langle\xi_{\circ}, \mathfrak{n}_{\circ}\right\rangle}+O\left(\left(\varepsilon^{2}+\epsilon_{\circ}\right)|x-\tilde{x}|\right) \\
& =s|x-\tilde{x}|\left(\left\langle\xi_{\circ}, \mathfrak{n}_{\circ}\right\rangle+\frac{1}{\left\langle\xi_{\circ}, \mathfrak{n}_{\circ}\right\rangle}\right)+O\left(\left(\varepsilon+\epsilon_{\circ}\right) \| x-\tilde{x} \mid\right) .
\end{aligned}
$$

Now $\left|\left\langle\xi_{\circ}, \mathfrak{n}_{\circ}\right\rangle\right| \geq c>0$ which is a consequence of the homogeneity relation $\rho(\xi)=\langle\xi, \nabla \rho(\xi)\rangle$. Hence, $|\langle x-\tilde{x}, \Xi(u)\rangle+t-\tilde{t}| \gtrsim|x-\tilde{x}|$ if $\varepsilon$ and $\epsilon_{\circ}$ are small enough, and another integration by parts in $\rho$ gives $(36)$.

Acknowledgement. The second named author would like to thank Fedya Nazarov.

\section{REFERENCES}

[1] M. Bézard, Une version générale de l'inégalité de Strichartz, C. R. Acad. Sci. Paris Sér. I Math. 315 (1992), no. 12, 1241-1244.

[2] J.Bourgain and L.Guth, Bounds on oscillatory integral operators based on multilinear estimates, Geom. Funct. Anal., to appear.

[3] L. Carleson and P. Sjölin, Oscillatory integrals and a multiplier problem for the disc, Studia Math. 44 (1972), 287-299.

[4] J.J. Duistermaat, Fourier integral operators. Progress in Mathematics, 130. Birkhäuser, 1996.

[5] G. Garrigós, A. Seeger, W. Schlag, Improvements in Wolff inequality for decompositions of cone multipliers. Manuscript.

[6] Y. Heo, F. Nazarov and A. Seeger, Radial Fourier multipliers in high dimensions, Acta Math. 206, (2011), no.1, 55-92.

[7] Y. Heo and C. Yang, Local smoothing estimates for some half-wave operators, J. Math. Anal. Appl., 381 (2011), 573-581.

[8] L. Hörmander, Fourier integral operators I, Acta Math. 127 (1971), 79-183.

[9] L. Hörmander, The analysis of linear partial differential operators Vols. I-IV, Springer-Verlag, Berlin, 1983, 1985.

[10] L. Hörmander, Oscillatory integrals and multipliers on $F L^{p}$, Ark. Mat. 11 (1971), 1-11.

[11] L. V. Kapitanskiǔ, Estimates for norms in Besov and Lizorkin-Triebel spaces for solutions of secondorder linear hyperbolic equations, (Russian) Zap. Nauchn. Sem. Leningrad. Otdel. Mat. Inst. Steklov. (LOMI) 171 (1989), Kraev. Zadachi Mat. Fiz. i Smezh. Voprosy Teor. Funktsii. 20, 106-162, 185-186; translation in J. Soviet Math. 56 (1991), no. 2, 2348-2389.

[12] I. Łaba and T. Wolff. A local smoothing estimate in higher dimensions, J. Anal. Math. 88 (2002), 149-171.

[13] S. Lee, Linear and bilinear estimates for oscillatory integral operators related to restriction to hypersurfaces, J. Funct. Anal. 241 (2006), no. 1, 56-98. 
[14] G. Mockenhaupt, A. Seeger, C.D. Sogge, Local smoothing of Fourier integral operators and CarlesonSjölin estimates. J. Amer. Math. Soc. 6 (1993), no. 1, 65-130.

[15] M. Pramanik, K.M. Rogers, A. Seeger, A Calderón-Zygmund estimate with applications to generalized Radon transforms and Fourier integral operators, Studia Math. 202 (2011), 1-15.

[16] A. Seeger, C.D. Sogge and E.M. Stein, Regularity properties of Fourier integral operators, Annals of Math. 134 (1991) 231-251.

[17] C.D. Sogge, Propagation of singularities and maximal functions in the plane, Invent. Math. 104 (1991), no. 2, 349-376.

[18] E. M. Stein, Oscillatory integrals in Fourier analysis, Beijing Lectures in Harmonic Analysis, Princeton Univ. Press, Princeton, N.J. (1986), 307-356.

[19] H. Triebel, Theory of function spaces. Monographs in Mathematics, 78. Birkhäuser Verlag, Basel, 1983.

[20] T. Wolff, Local smoothing type estimates on $L^{p}$ for large p, Geom. Funct. Anal. 10 (2000), no. 5, $1237-1288$.

Sanghyuk Lee, School of Mathematical Sciences, Seoul National University, Seoul 151742 , KOREA

E-mail address: shlee@math.snu.ac.kr

Andreas Seeger, Department of Mathematics, University of Wisconsin, 480 Lincoln Drive, MAdison, WI, 53706, USA

E-mail address: seeger@math.wisc.edu 